\title{
Octogenarians with blunt splenic injury: not all geriatrics are the same
}

\author{
Rame Bashir $^{1} \cdot$ Areg Grigorian $^{1} \cdot$ Michael Lekawa $^{1} \cdot$ Victor Joe $^{1} \cdot$ Sebastian D. Schubl ${ }^{1}$ Theresa L. Chin ${ }^{1}$. \\ Allen Kong ${ }^{1} \cdot$ Jeffry Nahmias ${ }^{1}$
}

Received: 8 July 2019 / Accepted: 11 April 2020 / Published online: 18 April 2020

(C) Italian Society of Surgery (SIC) 2020

\begin{abstract}
Geriatric trauma patients (GTP) (age $\geq 65$ years) with blunt splenic injury (BSI) have up to a $6 \%$ failure rate of non-operative management (NOM). GTPs failing NOM have a similar mortality rate compared to GTPs managed successfully with NOM. However, it is unclear if this remains true in octogenarians (aged 80-89 years). We hypothesized that the failure rate for NOM in octogenarians would be similar to their younger geriatric cohort, patients aged 65-79 years; however risk of mortality in octogenarians who fail NOM would be higher than that of octogenarians managed successfully with NOM. The Trauma Quality Improvement Program (2010-2016) was queried for patients with BSI. Those undergoing splenectomy within $6 \mathrm{~h}$ were excluded to select for patients undergoing NOM. Patients aged 65-79 years (young GTPs) were compared to octogenarians. A multivariable logistic regression model was used to determine the risk for failed NOM and mortality. From 43,041 BSI patients undergoing NOM, 3660 (8.5\%) were aged 65-79 years and 1236 (2.9\%) were octogenarians. Both groups had a similar median Injury Severity Score (ISS) $(p=0.10)$ and failure rate of NOM (6.6\% young GTPs vs. 6.8\% octogenarians $p=0.82)$. From those failing NOM, octogenarians had similar units of blood products transfused $(p>0.05)$ and a higher mortality rate $(40.5 \%$ vs. $18.2 \%, p<0.001)$, compared to young GTPs. Independent risk factors for failing NOM in octogenarians included $\geq 1$ unit of packed red blood cells (PRBC) $(p=0.039)$ within $24 \mathrm{~h}$ of admission. Octogenarians who failed NOM had a higher mortality rate compared to octogenarians managed successfully with NOM ( $40.5 \%$ vs $23.6 \% p=0.001)$, which persisted in a multivariable logistic regression analysis (OR 2.25, CI 1.37-3.70, $p<0.001$ ). Late failure of NOM $\geq 24 \mathrm{~h}$ (vs. early failure) was not associated with increased risk of mortality $(p=0.88)$, but $\geq 1$ unit of PRBC transfused had higher risk (OR 1.88, CI 1.20-2.95, $p=0.006$ ). Compared to young GTPs with BSI, octogenarians have a similar rate of failed NOM. Octogenarians with BSI who fail NOM have over a twofold higher risk of mortality compared to those managed successfully with NOM. PRBC transfusion increases risk for mortality. Therefore, clinicians should consider failure of NOM earlier in the octogenarian population to mitigate the risk of increased mortality.
\end{abstract}

Keywords Blunt splenic injury · Geriatric trauma patients · Octogenarian non-operative management

\section{Introduction}

The geriatric population, defined as those $\geq 65$ years old, compose $15 \%$ of the entire US population or roughly 46 million people [1]. By 2040, this number is expected to grow to $22 \%$ [1]. Medical advances have led to an increase in life expectancy and enable an active lifestyle into late adulthood,

Rame Bashir

ramebashir@gmail.com

1 Division of Trauma, Burns and Surgical Critical Care, Department of Surgery, University of California, Irvine Medical Center, 333 The City Blvd West, Suite 1600, Orange, CA 92868-3298, USA including driving at an older age [2]. As such, injury patterns in this population have shifted with trauma centers noting an increase in geriatric trauma patients (GTPs) suffering blunt trauma [3]. Additionally, octogenarians, defined as those 80-89 years old, are presenting to hospitals at increasing rates. Ukkonen et al. found that patients $\geq 80$ years old account for $15 \%$ of all emergency room visits nationwide [4]. Management of octogenarians requires special considerations due to suppressed stress responses, impaired central nervous system, altered cardiovascular physiology, as well as overall increased comorbidities [5-9]. Furthermore, the decreased abdominal musculature in geriatric patients may increase their risk for blunt splenic injury (BSI) [10]. 
In blunt trauma, the spleen is the most commonly injured solid organ [11]. Non-operative management (NOM) for BSI was once considered a relative contraindication in geriatric patients with failure rates reported as high as 70\% [12-16]. More recently, however, these rates have been proven to be exaggerated. Trust et al. used the National Trauma Databank to find that GTPs had slightly higher, but comparable failure rates of NOM compared to younger adults ( $6 \%$ versus $4 \%)$. Furthermore, this study concluded that failure of NOM for BSI was not an independent risk factor for mortality in GTPs, suggesting NOM to be a safe option. This practice is further supported by the most recent Eastern Association of the Surgery of Trauma (EAST) guidelines regarding BSI where age is no longer considered a contraindication to NOM [17].

Given the aging population and growing number of octogenarians presenting to trauma centers, we sought to determine whether management of BSI for octogenarian trauma patients would be different from younger GTPs. We hypothesized that the failure rate for NOM in octogenarians with BSI would be similar to younger GTPs, aged 65-79 years. However, we hypothesized the risk of mortality in octogenarians who fail NOM to be higher than octogenarians managed successfully with NOM.

\section{Methods}

We performed a retrospective analysis of the Trauma Quality Improvement Program (TQIP) over a 7-year period from 2010 to 2016 to identify patients between 65 and 89 years, presenting with splenic injury after blunt trauma, defined by the International Classification of Diseases (ICD) version 9 diagnosis codes: 865.0-865.19. Patients undergoing splenectomy were identified using the ICD-9 procedure code, 41.5 . The American College of Surgeons collects high-quality data from individual trauma centers across the USA in the TQIP database. This provides risk-adjusted benchmarking to compare trauma centers and opportunities to conduct research on a larger population.

The primary outcome was failure of NOM defined as: splenectomy after $6 \mathrm{~h}$ post-injury. Those undergoing splenectomy within $6 \mathrm{~h}$ were excluded to avoid including patients who did not undergo NOM similar to a previous analysis of NOM in BSI [18]. Two groups were compared: patients 65-79 years old (younger GTP) and patients $80-89$ years old (octogenarians).

Patient demographic information was collected including pre-hospital comorbidities and the median systolic blood pressure (SBP) on arrival. Pre-hospital comorbidities included congestive heart failure (CHF), end-stagerenal disease (ESRD), smoking, diabetes, hypertension, myocardial infarction (MI), cerebrovascular accident
(CVA), cirrhosis and chronic obstructive pulmonary disease (COPD). The injury profile included the Injury Severity Score (ISS), Abbreviated Injury Scale (AIS) and mechanism of injury. AIS is a scoring system that classifies each injury by body region on a six-point scale. The sum of the squares of the highest AIS scores for the three most severely injured body regions equals the ISS. The ISS ranges from 1 to 75 and correlates most closely with morbidity and mortality [19]. The mechanism of injuries were also evaluated to compare GTPs to octogenarians, including falls, motor vehicle collisions (MVCs), pedestrian accidents, and motorcycle collisions (MCCs).

Other outcomes evaluated included total hospital length of stay (LOS), intensive care unit (ICU) LOS, ventilator days, and units of packed red blood cells (PRBC), platelets and fresh frozen plasma (FFP) transfused within $24 \mathrm{~h}$. Since blood transfusion was a significant risk factor for failing NOM, we performed a sub-analysis of octogenarians in which we stratified patients by units of blood products transfused. Late failure of NOM, defined as failure $24 \mathrm{~h}$ after presentation, was compared to early failure (6-24 h post-injury). In 2013, TQIP began collecting data for splenic angioembolization intervention on patients that failed NOM within the first $48 \mathrm{~h}$ of patient arrival to the emergency department. Thus, for this study's 2010-2016 population, evaluation of angioembolization was performed for only the 2013-2016 population.

Descriptive statistics were performed for all variables. Continuous variables were compared using a Mann-Whitney $U$ test, while categorical variables were compared using Chi square. Categorical data were reported as percentages, and continuous data were reported as medians with interquartile range or means with standard deviation. The magnitude of the association between covariates and risk of failing NOM was measured using a univariable logistic regression model. These variables were chosen based on a review of the literature and included hypotension (systolic blood pressure $\leq 90 \mathrm{mmHg}$ ) within $24 \mathrm{~h}$, ISS $\geq 25$, massive blood product transfusion ( $\geq 5$ units of packed red blood cells within 4-h), severe (grade $>3$ ) AIS for the head, spine, thorax, abdomen, history of CHF, ESRD, diabetes, hypertension, COPD, CVA, MI, and smoking [20,21]. Covariates were then controlled for using a hierarchical multivariable logistic regression model. The adjusted risk of failure of NOM and mortality were reported with an odds ratio (OR) and $95 \%$ confidence intervals $(\mathrm{CI})$. We also performed a multivariable regression analysis of octogenarians managed non-operatively for BSI, with the same covariates, to analyze the risk of mortality. All $p$ values were two-sided, with a statistical significance level of $<0.05$. All statistical analyses were performed with IBM SPSS Statistics for Windows, Version 24. (Armonk, NY: IBM Corp). 


\section{Results}

\section{Demographics of geriatric patients undergoing NOM for BSI}

From 43,041 patients undergoing NOM after BSI, 3,660 $(8.50 \%)$ were younger GTPs, and 1,236 (2.9\%) were octogenarians. Compared to the younger GTPs, octogenarians were less often male $(51.1 \%$ vs $60.1 \%, p<0.001)$ and had a lower rate of severe thorax injury (AIS $>3)(16.7 \%$ vs $19.4 \%, p=0.04)$, but similar ISS $(p=0.10)$. The younger GTP group had a higher rate of injury secondary to MCCs (5.8\% vs $0.0 \%, p=0.02)$, but a similar rate of falls $(40.8 \%$ vs $48.8 \%, p=0.16)$, MVCs $(42.6 \%$ vs $44.0 \% p=0.81)$, and pedestrian accidents $(6.6 \%$ vs $6.0 \% p=0.83)$ compared to the older octogenarian group. The octogenarian group had higher rates of $\mathrm{CHF}(10.5 \%$ vs $5.5 \%, p<0.001)$, hypertension $(59.2 \%$ vs $53.8 \%, p=0.001)$, and CVA ( $4.6 \%$ vs $3.0 \%, p=0.006)$, but lower rates of diabetes $(18.6 \%$ vs $24.1 \%, p<0.001)$ and history of smoking $(3.1 \%$ vs $10.3 \%$, $p<0.001)$ compared to the younger GTPs. The octogenarian group failed NOM at a similar rate compared to the younger GTP cohort (6.8\% vs 6.6\%, $p=0.82)$ (Table 1$)$.

\section{Analysis for risk of failure of NOM among geriatric BSI patients}

In a multivariable analysis, the strongest risk factors for failing NOM of BSI was an abdominal AIS $>3$ (OR 4.58 , CI $2.46-8.53, p<0.001$ ), followed by $\geq 5$ units of PRBC transfusions within $4 \mathrm{~h}$ of admission (OR 2.01, CI 1.13-3.60, $p=0.02$ ) (Table 2).

After adjusting for ISS $\geq 25$, octogenarians who received $\geq 1$ unit $P R B C$ transfusion within $24 \mathrm{~h}$ had a higher risk for failing NOM (OR 2.05, CI 1.01-4.11, $p=0.039$ ). Regarding FFP and platelets, there was a stepwise increased risk of failing NOM with an increased number of units transfused (Table 3 ).
Table 1 Demographics of blunt splenic injury with nonoperative management

\begin{tabular}{lccc}
\hline Characteristic & Age 65-79 $(n=3660)$ & Age $80-89(n=1236)$ & $p$ value \\
\hline Age, year, median (IQR) & $70.0(7)$ & $84.0(5)$ & $<0.001$ \\
Male, $n(\%)$ & $2197(60.1 \%)$ & $631(51.1 \%)$ & $<0.001$ \\
ISS, median (IQR) & $19.0(13)$ & $18.0(12)$ & 0.10 \\
Lowest SBP within 24 h, median (IQR) & $75.0(43)$ & $75.0(35)$ & 0.98 \\
Mechanism of trauma, $n(\%)$ & & & \\
Fall & $97(40.1 \%)$ & $41(48.8 \%)$ & 0.16 \\
Motor vehicle collision & $103(42.6 \%)$ & $37(44.0 \%)$ & 0.81 \\
Pedestrian & $16(6.6 \%)$ & $5(6.0 \%)$ & 0.83 \\
Motorcycle collision & $14(5.8 \%)$ & $0(0 \%)$ & 0.02 \\
Comorbidities, $n$ (\%) & & & \\
Congestive heart failure & $201(5.5 \%)$ & $130(10.5 \%)$ & 0.001 \\
End-stage renal disease & $60(1.6 \%)$ & $19(1.5 \%)$ & 0.81 \\
Smoker & $376(10.3 \%)$ & $38(3.1 \%)$ & $<0.001$ \\
Diabetes & $883(24.1 \%)$ & $230(18.6 \%)$ & 0.001 \\
Hypertension & $1968(53.8 \%)$ & $732(59.2 \%)$ & 0.001 \\
COPD & $456(12.5 \%)$ & $142(11.5 \%)$ & 0.37 \\
Myocardial infarction & $102(2.8 \%)$ & $30(2.4 \%)$ & 0.50 \\
Cerebrovascular accident & $109(3.0 \%)$ & $57(4.6 \%)$ & 0.006 \\
Cirrhosis & $63(1.7 \%)$ & $15(1.2 \%)$ & 0.22 \\
AIS (grade $>$ 3), $n(\%)$ & $627(17.1 \%)$ & $220(17.8 \%)$ & 0.59 \\
Head & $709(19.4 \%)$ & $207(16.7 \%)$ & 0.04 \\
Thorax & $678(18.5 \%)$ & $206(16.7 \%)$ & $84(6.8 \%)$ \\
Abdomen & $242(6.6 \%)$ & & \\
Failed non-operative management, $n(\%)$ & & \\
\hline
\end{tabular}

$I Q R$ interquartile range, $I S S$ Injury Severity Score, $S B P$ systolic blood pressure, $C O P D$ chronic obstructive pulmonary disease, AIS Abbreviated Injury Scale 
Table 2 Multivariable analysis of risk factors for failure of nonoperative management in blunt splenic injury

\begin{tabular}{llll}
\hline Risk factor & OR & CI & $p$ value \\
\hline Octogenarian (vs. age 65-79) & 1.49 & $0.79-2.83$ & 0.22 \\
ISS $\geq 25$ & 0.77 & $0.40-1.49$ & 0.44 \\
Hypotensive within 24 h & 1.00 & $0.55-1.82$ & 0.99 \\
PRBC transfusion $\geq 5$ units within 4h & 2.01 & $1.13-3.60$ & 0.02 \\
Abbreviated injury scale_head (grade $>3$ ) & 0.50 & $0.21-1.17$ & 0.11 \\
Abbreviated injury scale_thorax (grade $>3$ ) & 1.04 & $0.54-2.00$ & 0.92 \\
Abbreviated injury scale—abdomen (grade $>3$ ) & 4.58 & $2.46-8.53$ & $<0.001$ \\
Congestive heart failure & 0.84 & $0.28-2.50$ & 0.75 \\
End-stage renal disease & 0.71 & $0.08-5.99$ & 0.75 \\
Smoker & 1.80 & $0.73-4.42$ & 0.20 \\
Diabetes & 1.72 & $0.90-3.31$ & 0.10 \\
Hypertension & 0.91 & $0.51-1.62$ & 0.74 \\
COPD & 1.69 & $0.79-3.63$ & 0.18 \\
History of myocardial infarction & 1.48 & $0.30-7.18$ & 0.63 \\
History of cerebrovascular accident & 0.65 & $0.08-5.04$ & 0.68 \\
\hline
\end{tabular}

ISS Injury Severity Score, $P R B C$ packed red blood cells, $C O P D$ chronic obstructive pulmonary disease
Table 3 Multivariable analysis of risk factors for failure of non-operative management in octogenarians with blunt splenic injury (reference: no blood products transfused)

\begin{tabular}{lcll}
\hline Risk factor & OR & CI & $p$ value \\
\hline \multicolumn{2}{l}{ PRBC transfusion within $24 \mathrm{~h}$} & & \\
$\geq 1$ & 2.05 & $1.02-4.11$ & 0.039 \\
$\geq 2$ & 2.32 & $1.16-4.64$ & 0.019 \\
$\geq 3$ & 2.04 & $1.01-4.32$ & 0.041 \\
$\geq 4$ & 1.92 & $0.88-4.22$ & 0.042 \\
FFP transfusion within $24 \mathrm{~h}$ & & \\
$\geq 1$ & 2.41 & $1.18-4.93$ & 0.016 \\
$\geq 2$ & 2.42 & $1.15-5.07$ & 0.019 \\
$\geq 3$ & 3.00 & $1.34-6.69$ & 0.007 \\
$\geq 4$ & 3.83 & $1.70-8.64$ & 0.001 \\
Platelets transfusion & within $24 \mathrm{~h}$ & & \\
$\geq 1$ & 1.99 & $0.84-4.71$ & 0.119 \\
$\geq 2$ & 3.94 & $1.59-9.76$ & 0.003 \\
$\geq 3$ & 3.83 & $1.19-12.37$ & 0.025 \\
$\geq 4$ & 6.25 & $1.47-26.63$ & 0.013 \\
\hline
\end{tabular}

Each model controlled for Injury Severity Score $\geq 25$

$P R B C$ packed red blood cells, FFP fresh frozen plasma

\section{Analysis for risk of mortality among octogenarian undergoing NOM BSI patients}

Among the patients who failed NOM of BSI, octogenarians had a higher incidence of mortality ( $40.5 \%$ versus $18.2 \%$, $p<0.001$ ), compared to the younger GTPs (Table 4). Beginning in 2013, octogenarians were more likely to require splenic angioembolization within the first $48 \mathrm{~h}$ of arriving at the emergency department $(12.0 \%$ vs $2.7 \%, p=0.01)$, compared to the younger GTPs (Table 4). After adjusting for covariates, octogenarians continued to have a higher risk of mortality compared to the younger geriatric cohort after failing NOM (OR 4.99, CI 1.11-22.49, $p=0.04$ ) (Table 5). The strongest risk factor of mortality among octogenarians with BSI managed non-operatively, in descending order, were ISS $\geq 25$ (OR 3.80, CI 2.81-5.13, $p<0.001$ ), thoracic injury with an AIS $>3$ (OR 2.13, CI 1.50-3.03, $p<0.001$ ), and $\mathrm{PRBC}$ transfusion $\geq 1$ unit within $24 \mathrm{~h}$ (OR 1.88, CI $1.20-2.95, p=0.006$ ). Late failure of NOM was not found to be associated with increased risk of mortality among octogenarians (OR 0.92, CI 0.32-2.68, $p=0.88$ ) (Table 6).

\section{Discussion}

This is a large multi-year contemporary national analysis of GTPs that stratifies the GTP population by age to compare the outcomes of octogenarians to their younger geriatric cohort undergoing NOM of BSI. Our data suggest that GTPs were more likely to suffer from MCCs, but had similar rates of falls, MVCs and pedestrian accidents. Octogenarians fail at similar rates to younger GTPs, but when octogenarians fail, they have a fivefold higher risk of mortality, compared to their younger geriatric cohort. Among the octogenarian subgroup, those failing NOM are at a twofold higher risk of mortality compared to those managed successfully with NOM, even after adjusting for ISS. Furthermore, any octogenarian receiving $\geq 1$ unit $\mathrm{PRBC}$ transfusion is at an increased risk of failing NOM.

The failure rate of GTPs undergoing NOM of BSI is relatively low. In recent studies, failure rates range from $4.6 \%$ to $6 \%[12,18]$. These rates are significantly improved 
Table 4 Demographics and outcomes of blunt splenic injury failing non-operative management

\begin{tabular}{|c|c|c|c|}
\hline Characteristic & Age $65-79(n=242)$ & Age $80-89(n=84)$ & $p$ value \\
\hline Age, year, median (IQR) & $71.0(7)$ & $83.0(4)$ & $<0.001$ \\
\hline ISS, median (IQR) & $22.0(14)$ & $22.0(11)$ & 0.58 \\
\hline Lowest SBP within $24 \mathrm{~h}$, median (IQR) & $84.0(37)$ & $75.0(26)$ & 0.26 \\
\hline \multicolumn{4}{|l|}{ AIS (grade $>3), n(\%)$} \\
\hline Head & $30(12.4 \%)$ & $1(1.2 \%)$ & 0.003 \\
\hline Thorax & $38(15.7 \%)$ & $10(11.9 \%)$ & 0.40 \\
\hline Abdomen & $109(45.0 \%)$ & $37(44.0 \%)$ & 0.88 \\
\hline LOS, days, median (IQR) & $14.0(14)$ & $11.0(11)$ & 0.01 \\
\hline ICU, days, median (IQR) & $8.0(12)$ & $6.0(10)$ & 0.41 \\
\hline Ventilator, days, median (IQR) & $7.0(15)$ & $5.0(9)$ & 0.17 \\
\hline Angioembolization within $48 \mathrm{~h}, n(\%)$ & $4(2.7 \%)$ & $6.0(12.0 \%)$ & 0.01 \\
\hline \multicolumn{4}{|c|}{ Blood product transfusion units within $24 \mathrm{~h}$, median (IQR) } \\
\hline Packed red blood cells & $5.0(9)$ & $8.0(13)$ & 0.98 \\
\hline Fresh frozen plasma & $2.0(6)$ & $4.0(11)$ & 0.91 \\
\hline Platelets & $1.0(2)$ & $2.0(4)$ & 0.42 \\
\hline \multicolumn{4}{|l|}{ Complications, $n(\%)$} \\
\hline Acute kidney injury & $16(6.6 \%)$ & $9(10.7 \%)$ & 0.22 \\
\hline ARDS & $15(6.2 \%)$ & $3(3.6 \%)$ & 0.36 \\
\hline Unplanned ICU admission & $27(11.2 \%)$ & $4(4.8 \%)$ & 0.09 \\
\hline Unplanned intubation & $33(13.6 \%)$ & $8(9.5 \%)$ & 0.33 \\
\hline Deep vein thrombosis & $15(6.2 \%)$ & $4(4.8 \%)$ & 0.63 \\
\hline Pulmonary embolism & $7(2.9 \%)$ & 0 & 0.12 \\
\hline Pneumonia/VAP & $43(17.8 \%)$ & $10(11.9 \%)$ & 0.21 \\
\hline Mortality, $n(\%)$ & $44(18.2 \%)$ & $34(40.5 \%)$ & $<0.001$ \\
\hline
\end{tabular}

$I Q R$ interquartile range, ISS Injury Severity Score, $S B P$ systolic blood pressure, AIS abbreviated injury scale, $L O S$ length of stay, $I Q R$ interquartile range, ICU intensive care unit, $P R B C$ packed red blood cells, $F F P$ fresh frozen plasma, $A R D S$ acute respiratory distress syndrome, VAP ventilator associated pneumonia
Table 5 Multivariable analysis of risk factors for mortality among trauma patients failing non-operative management in blunt splenic injury

\begin{tabular}{llll}
\hline Risk factor & OR & CI & $p$ value \\
\hline Octogenarian (vs. age 65-79) & 4.99 & $1.11-22.49$ & 0.04 \\
ISS $\geq 25$ & 2.52 & $0.61-10.52$ & 0.20 \\
Hypotensive within 24 h & 5.69 & $0.98-33.02$ & 0.05 \\
Abbreviated injury scale—tho- & 3.73 & $0.78-17.86$ & 0.10 \\
$\quad$ rax (grade $>$ 3) & & & \\
Congestive heart failure & 0.33 & $0.03-4.47$ & 0.41 \\
Smoker & 0.70 & $0.08-6.51$ & 0.75 \\
Diabetes & 0.78 & $0.13-4.58$ & 0.79 \\
Hypertension & 0.80 & $0.20-3.13$ & 0.75 \\
COPD & 1.69 & $0.79-3.63$ & 0.18 \\
\hline
\end{tabular}

ISS Injury Severity Score, COPD chronic obstructive pulmonary disease

from prior studies in the early 2000s with failure rates of $11-33 \%$ [14, 22-26]. Our data parallel the most recent studies demonstrating an overall low failure rate among the general geriatric population, less than $7 \%$ for patients between
Table 6 Multivariable logistic regression analysis for risk of mortality in octogenarians with blunt splenic injury managed non-operatively

\begin{tabular}{lllr}
\hline Risk factor & OR & CI & $p$ value \\
\hline Failed NOM & 2.25 & $1.37-3.70$ & 0.001 \\
Failed NOM $\geq 24 \mathrm{~h}(\mathrm{vs}<24 \mathrm{~h})$ & 0.92 & $0.32-2.68$ & 0.88 \\
ISS $\geq 25$ & 3.80 & $2.81-5.13$ & $<0.001$ \\
PRBC transfusion $\geq 1$ unit within $24 \mathrm{~h}$ & 1.88 & $1.20-2.95$ & 0.006 \\
Abbreviated injury scale—-thorax & 2.13 & $1.50-3.03$ & $<0.001$ \\
$\quad$ grade $>3$ ) & & & \\
Congestive heart failure & 1.59 & $1.02-2.49$ & 0.04 \\
Smoker & 1.12 & $0.49-2.57$ & 0.80 \\
Diabetes & 1.18 & $0.81-1.72$ & 0.40 \\
Hypertension & 0.52 & $0.39-0.69$ & $<0.001$ \\
History of cerebrovascular accident & 1.25 & $0.65-2.41$ & 0.50 \\
\hline
\end{tabular}

NOM non-operative management, ISS Injury Severity Score, $P R B C$ packed red blood cells

65-89 years of age. In addition, we found that octogenarians have a similar rate and risk of failure of NOM of BSI compared to the younger geriatric cohort, despite higher rates of 
some comorbidities such as $\mathrm{CHF}$ and hypertension. Our data suggest risk factors for octogenarians failing NOM include receiving $\geq 1$ unit of PRBC, FFP, or platelet transfusions within $4 \mathrm{~h}$. Both FFP and platelet transfusion increase the risk of failure in a stepwise fashion with each additional unit transfused. While it is certain that the transfusion occurs because of the failure of NOM, it suggests that there is no safe level of transfusion requirement in the octogenarian population, as even one unit of transfusion is associated with failure of NOM.

Octogenarians failing NOM of BSI are susceptible to a higher rate of mortality. Geriatric patients failing NOM previously had been demonstrated to have no difference in mortality by Albrecht et al. [14]. Similarly, Trust et al. concluded that no association exists between failing NOM and mortality among GTPs aged 60-89 years [18]. However, our data augment the findings of Trust et al. by illustrating a fivefold increased risk of mortality among octogenarians failing NOM. In addition, we identified other risk factors for mortality among octogenarians failing NOM: specifically, one unit or more of PRBC transfusion. This data suggests that when performing NOM for BSI in octogenarians, the managing physician should have a lower threshold for operation, as waiting to operate until a single unit of blood is transfused may be associated with increased mortality in this population.

As a retrospective database study, there are inherent limitations including reporting bias and coding errors. Furthermore, there are pertinent missing variables including the severity of splenic injury (e.g., American Association for the Surgery of Trauma grade). The use of abdominal AIS is the closest representation to the degree of splenic injury and is tracked by the database; however, concomitant abdominal injuries may account for different AIS scores, thereby this measure may not accurately reflect the degree of injury to the spleen and thus may introduce bias to the study. Other missing pertinent variables include information regarding coagulopathy (i.e., use of antiplatelet or anticoagulants), anemia, and what was the actual clinical trigger for operation. Also, angioembolization intervention was only assessed for half our study population, because TQIP did not begin collecting this data until 2013. This prevented our ability to analyze our entire population for the 2010-2016 period of this study; however, our small sample size of data demonstrated increased use of angioembolization in octogenarians which merits future study. In addition, TQIP does not provide ongoing physiologic data or physical examination data (e.g., development of peritonitis). Despite these limitations, this is the first evaluation of octogenarians using a large trauma database over multiple years. We found that octogenarians with more severe injuries and higher grade BSI are at a higher risk of NOM failure and mortality compared to a generalized GTP cohort. Furthermore, our study is strengthened by its generalizability as TQIP encompasses over 800 centers.

\section{Conclusion}

Compared to younger GTPs with BSI, octogenarians have a similar rate and risk for failure of NOM, which reaffirms that age should not be a determinant for performance of NOM for BSI. However, octogenarians with BSI who fail NOM have over a twofold higher risk of mortality compared to those managed successfully with NOM and a greater than fivefold higher risk than younger GTPs that fail NOM. Furthermore, one or more units of PRBC transfused increases the risk for mortality in this octogenarian cohort and therefore octogenarians should be considered for splenectomy at a lower threshold, even prior to the need for transfusion if possible.

Funding This research did not receive any specific grant from funding agencies in the public, commercial, or not-for-profit sectors.

\section{Compliance with ethical standards}

Conflict of interest The authors report no conflicts of interest, financial or otherwise.

Research involving human participants and/or animals This article does not contain any studies with animals performed by any of the authors.

Informed consent For this type of article, informed consent is not required.

\section{References}

1. Ortman J, Velkoff V, Hogan H (2014) An aging nation: the older population in the United States. U.S. Department of Commerce Economics and Statistics Administration

2. Ikpeze TC, Elfar JC (2016) The geriatric driver: factors that influence when to stop driving. Geriatr Orthop Surg Rehabil 7(2):106-109

3. Brown CV, Rix K, Klein AL, Ford B, Teixeira PG, Aydelotte J, Ali S (2016) A comprehensive investigation of comorbidities, mechanisms, injury patterns, and outcomes in geriatric blunt trauma patients. Am Surg 82(11):1055-1062

4. Ukkonen M, Jämsen E, Zeitlin R et al (2019) Emergency department visits in older patients: a population-based survey. BMC Emerg Med 19:20

5. Cerreta F, Eichler HG, Rasi G (2012) Drug policy for an aging population-the European Medicines Agency's geriatric medicines strategy. N Engl J Med 367(21):1972

6. Lustenberger T, Talving P, Schnüriger B, Eberle BM, Keel MJ (2012) Impact of advanced age on outcomes following damage control interventions for trauma. World J Surg 36(1):208

7. Demetriades D, Sava J, Alo K et al (2001) Old age as a criterion for trauma team activation. J Trauma 51:754-756 
8. Jacobs DG (2003) Special considerations in geriatric injury. Curr Opin Crit Care 9(6):535-539

9. Brooks SE, Peetz AB (2017) Evidence-based care of geriatric trauma patients. Surg Clin N Am 97(5):1157-1174

10. Nishijima DK, Simel DL, Wisner DH, Holmes JF (2012) Does this adult patient have a blunt intra-abdominal injury? JAMA 307(14):1517-1527. https://doi.org/10.1001/jama.2012.422

11. Yorkgitis BK (2017) Primary care of the blunt splenic injured adult. Am J Med 130(3):365.e1-365.e5

12. Siriratsivawong K, Zenati M, Watson GA, Harbrecht BG (2007) Nonoperative management of blunt splenic trauma in the elderly: does age play a role? Am Surg 73(6):585

13. Cocanour CS, Moore FA, Ware DN et al (2000) Age should not be a consideration for nonoperative management of blunt splenic injury. J Trauma 48:606-610

14. Albrecht RM, Schermer CR, Morris A (2002) Nonoperative management of blunt splenic injuries: factors influencing success in age \%3e55 years. Am Surg 68:227-230

15. Myers JG, Dent DL, Stewart RM et al (2000) Blunt splenic injuries: dedicated trauma surgeons can achieve a high rate of nonoperative success in patients of all ages. J Trauma 48:801-805

16. Watson GA, Rosengart MR, Zenati MS, Tsung A, Forsythe RM, Peitzman AB, Harbrecht BG (2006) Nonoperative management of severe blunt splenic injury: are we getting better? J Trauma 61(5):1113-1118 (discussion 1118)

17. Stassen N, Bhullar I, Cheng J, Crandall M, Friese R, Guillamondegui O, Kerwin A (2012) Selective nonoperative management of blunt splenic injury: an Eastern Association for the Surgery of Trauma practice management guideline. J Trauma Acute Care Surg 73(5 Suppl 4):S294-S300

18. Trust MD, Teixeira PG, Brown LH, Ali S, Coopwood B, Aydelotte JD, Brown CVR (2018) Is it safe? Nonoperative management of blunt splenic injuries in geriatric trauma patients. J Trauma Acute Care Surg 84(1):123-127

19. Javali RH, Krishnamoorthy AP, Srinivasarangan M, Suraj S (2019) Comparison of injury severity score, new injury severity score, revised trauma score and trauma and injury severity score for mortality prediction in elderly trauma patients. Indian J Crit Care Med 23(2):73-77. https://doi.org/10.5005/jp-journals-10071 $-23120$

20. Ochsner MG (2001) Factors of failure for nonoperative management of blunt liver and splenic injuries. World J Surg 25:1393-1396

21. Sanders MN, Civil I (1999) Adult splenic injuries: treatment patterns and predictive indicators. Aust N Z J Surg 69:430-432

22. Peitzman AB, Harbrecht BG, Rivera L, Heil B (2005) Failure of observation of blunt splenic injury in adults: variability in practice and adverse consequences. J Am Coll Surg 201-2:179-187

23. Bee TK, Croce MA, Miller PR, Pritchard FE, Fabian TC (2001) Failures of splenic nonoperative management: is the glass half empty or half full? J Trauma 50(2):230-236

24. Harbrecht BG, Peitzman AB, Rivera L, Heil B, Croce M, Morris JA, Enderson BL, Kurek S, Pasquale M, Frykberg ER et al (2001) Contribution of age and gender to outcome of blunt splenic injury in adults: multicenter study of the eastern association for the surgery of trauma. J Trauma 51(5):887-895

25. Nix JA, Costanza M, Daley BJ, Powell MA, Enderson BL (2001) Outcome of the current management of splenic injuries. J Trauma 50(5):835-842

26. Cocanour CS, Moore FA, Ware DN, Marvin RG, Duke JH (2000) Age should not be a consideration for nonoperative management of blunt splenic injury. J Trauma 48(4):606-610 (discussion 610-612)

Publisher's Note Springer Nature remains neutral with regard to jurisdictional claims in published maps and institutional affiliations. 